\title{
SEOM clinical guidelines in Hereditary Breast and ovarian cancer
}

\author{
G. Llort ${ }^{1} \cdot$ I. Chirivella ${ }^{2} \cdot$ R. Morales ${ }^{3} \cdot$ R. Serrano ${ }^{4}$ A. Beatriz Sanchez ${ }^{5}$.

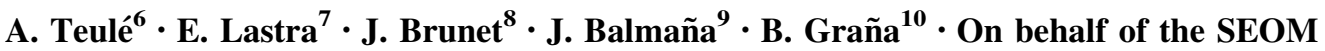 \\ Hereditary Cancer Working Group
}

Received: 13 October 2015/Accepted: 13 October 2015/Published online: 15 December 2015

(C) The Author(s) 2015. This article is published with open access at Springerlink.com

\begin{abstract}
Approximately, $7 \%$ of all breast cancers (BC) and $11-15 \%$ of ovarian cancers (OC) are associated with inherited predisposition, mainly related to germline mutations in high penetrance BRCA1/2 genes. Clinical criteria for genetic testing are based on personal and family history to estimate a minimum $10 \%$ detection rate. Selection criteria are evolving according to new advances in this field and the clinical utility of genetic testing. Multiplex panel testing carries its own challenges and we recommend inclusion of genes with clinical utility. We recommend screening with annual mammography from age 30 and breast MRI from age 25 for BRCA1 and BRCA2 mutation carriers. Bilateral salpingo-oophorectomy should be offered to women with a BRCA1 or BRCA2 mutation, between 35 and 40 years and after completion of childbearing, or individualise based on the earliest age of ovarian cancer diagnosed in the family. Bilateral risk-reducing mastectomy is an option for healthy BRCA1 and
\end{abstract}

G. Llort

gllort@cst.cat

1 Unidad de Consejo Genético, Servicio de Oncología Médica, Corporació Sanitària Parc Taulí/Consorci Sanitari de Terrassa, Parc Taulí s/n, 08208 Sabadell, Barcelona, Spain

2 Oncología Médica, Hospital Clínico Universitario de Valencia, Valencia, Spain

3 Servicio de Oncología Médica, Hospital La Mancha Centro, Ciudad Real, Spain

4 Unidad de Consejo Genético, Servicio de Oncología Médica, Hospital Universitario Reina Sofía (Córdoba), Córdoba, Spain

5 Unidad de Consejo Genético en Cáncer, Servicio de Oncología Médica, Hospital General Universitario de Elche, Alicante, Spain
BRCA2 mutation carriers, as well as contralateral mastectomy for young patients with a prior $\mathrm{BC}$ diagnosis. BRCA genetic testing in patients with $\mathrm{BC}$ and $\mathrm{OC}$ may influence their locoregional and systemic treatment.

Keywords Hereditary breast and ovarian cancer . BRCA1 and BRCA2 genes - Prevention - SEOM

\section{Hereditary breast and ovarian cancer syndrome (HBOC): introduction}

Approximately, $7 \%$ of all breast cancers (BC) and $11-15 \%$ of ovarian cancers (OC) are associated with inherited predisposition, mainly related to germline mutations in high penetrance BRCA1/2 genes. A meta-analysis reports mean cumulative $\mathrm{BC}$ risk at age 70 years of $57 \%$ (95\% CI 47-66) for BRCAl and $49 \%$ (95\% CI 40-57)

6 Unidad de Consejo Genético, Programa de Cáncer Hereditario, Institut Català d'Oncologia-IDIBELL, L'Hospitalet del Llobregat, Barcelona, Spain

7 Unidad de Consejo Genético, Sección Oncología Médica, Hospital Universitario de Burgos, Burgos, Spain

8 Unidad de Consejo Genético, Programa de Cáncer Hereditario, Institut Català d'Oncologia-IDIBGI, Girona, Dpto Ciencias Médicas, Facultad de Medicina, Universidad de Girona, Girona, Spain

9 Programa Cáncer Familiar, Servicio Oncología Médica, Hospital Vall d'Hebron, Barcelona, Spain

10 Unidad de Alto riesgo en Cáncer, Servicio de Oncologia Médica, Complejo Hospitalario Universitario A Coruña (CHUAC), La Coruña, Spain 
for $B R C A 2$ mutation carriers; and OC risk of $40 \%(95 \%$ CI 35-46) for BRCA1 and $18 \%$ (95\% CI 13-23) for BRCA2 mutation carriers [1, 2].

Advances in sequencing technologies make massive parallel sequencing more feasible and afford testing for other hereditary predisposition genes assigned to high $\mathrm{BC}$ risk (TP53, PALB2, PTEN), moderate BC risk (CHEK2, $A T M, N F 1, N B N)$, elevated, but imprecise $\mathrm{BC}$ risk $(C D H 1$, STK11) and OC risk (MMR genes, RAD51D, BRIP1) [3].

HBOC families associated to BRCA1 or BRCA2 germline mutations present an autosomal dominant hereditary pattern, with early ages of cancer onset, bilaterality and male breast cancer. BRCAI-associated BC usually have a higher histological grade and a triple-negative basal phenotype. OC in BRCA1 and BRCA2 mutation carriers are high-grade serous adenocarcinoma with intraepithelial infiltrates, lymphocytic atypia and abundant mitoses.

\section{Criteria for BRCA genetic testing}

We recommend genetic counselling pre- and post-germline genetic testing. Genetic counselling is a process that guarantees a discussion about the benefits and limitations of genetic testing, provides risk estimates of developing cancer, recommendations for early detection and preventive measures, information about reproductive options and support for psychological well-being.

Clinical criteria for genetic testing are based on personal and family history to estimate a minimum $10 \%$ detection rate $[4,5]$. Hence, selection criteria are evolving according to new advances in this field and the clinical utility of genetic testing (Table 1). Multiplex panel testing carries its own challenges and we recommend inclusion of genes with clinical utility. If multiplex testing is considered for $\mathrm{HBOC}$, we recommend including TP53, PALB2, BRIP1, RAD51C and RAD51D [6]. Other genes like $C D H 1$ and PTEN might be offered based on familial phenotype (bilateral lobular $\mathrm{BC}<50$, Cowden-like features) or when specific criteria for the hereditary cancer syndrome are present.

\section{Surveillance and strategies for early detection of cancer in mutation carriers}

Early detection of breast cancer aims to reduce morbidity and mortality. An individual patient data meta-analysis of high-quality observational studies shows that the use of breast MRI as an adjunct to mammography significantly increases the sensitivity of screening in women with BRCA1 and BRCA2 mutations as compared with mammography alone (93.4 vs $39.6 \% ; p<0.001)$, whereas specificity is significantly reduced $(80.3$ vs $93.6 \%$; $p=0.0016$ ) [7]. Although a survival benefit from breast MRI in these women has not robustly been proven, a clear trend towards improved metastasis-free survival has been reported [8]. Therefore, we recommend annual mammography and breast MRI screening for BRCA1 and BRCA2 mutation carriers (IIA).

The appropriateness of imaging scheduling is still controversial. The pooled-data analysis includes women between 30 and 70 years, with MRI and mammography being performed within 1-2 months from each other [7]. In addition, radiation-induced breast cancer is a potential concern in women younger than 30 years, who also present denser breast tissue which hampers a good visualisation on mammograms. So, we recommend an annual breast MRI from age 25 , with a synchronous annual mammography added after age 30 until age 70 (Table 2).

There are limited data to support breast imaging in men, but we recommend considering mammography at age 40 years, especially if gynaecomastia or in BRCA2 carriers (IIIC).

Women with a $B R C A 1$ or $B R C A 2$ mutation who have not chosen prophylactic salpingo-oophorectomy may follow determination of $\mathrm{Ca} 125$ and transvaginal ultrasound since age 35 (IIC), but they should be informed that early detection of ovarian cancer is not guaranteed.

Screening for prostate cancer at age 40 is recommended for males with a BRCA2 mutation, due to the increased risk and poor survival outcomes, and should be individualised for BRCA1 male mutation carriers [9] (IIB).

Screening for colorectal cancer with annual FIT or colonoscopy every 5 years beginning at 40 should be encouraged especially in BRCAl carriers (IIIC).

Consider individualised screening for pancreatic cancer and melanoma, based on family history.

\section{Risk-reducing surgery}

\section{Bilateral salpingo-oophorectomy}

The absence of reliable methods for early detection and the poor prognosis associated with advanced ovarian cancer have supported the recommendation of bilateral risk reduction salpingo-oophorectomy (RRSO) after completion of childbearing in women with BRCA1 or BRCA2 mutation [10]. Preventive oophorectomy was associated with an $80 \%$ reduction in the risk of ovarian, fallopian tube or peritoneal cancer in $B R C A 1$ or $B R C A 2$ carriers and a $77 \%$ reduction in all-cause mortality $[10,11]$.

A $1-4.3 \%$ residual risk of a primary peritoneal carcinoma has been reported in some studies [12]. A metaanalysis involving 10 studies showed an approximately $80 \%$ reduction in the risk of ovarian or fallopian cancer 
Table 1 Selection criteria for $B R C A$ genetic testing

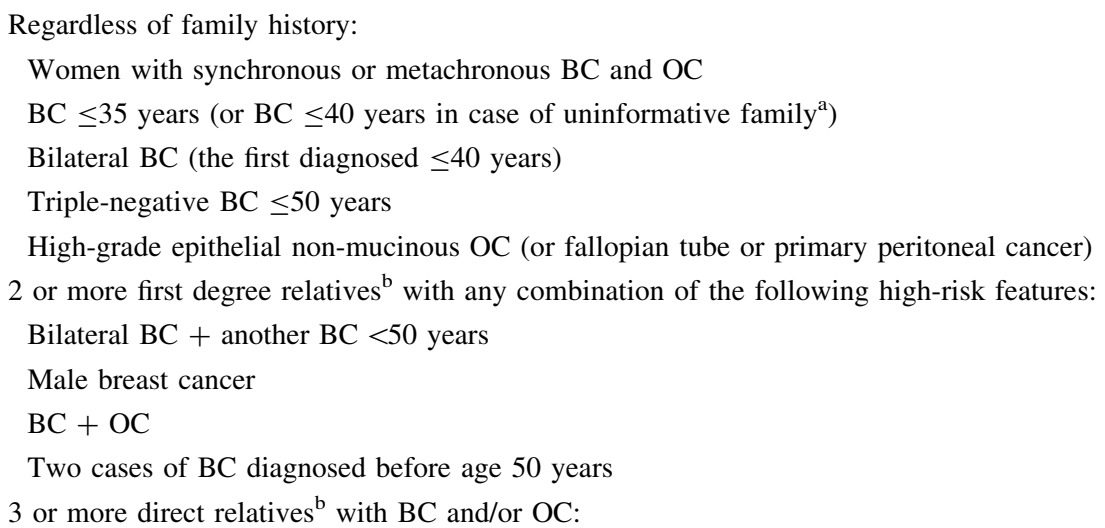

Regardless of family history:

Women with synchronous or metachronous $\mathrm{BC}$ and $\mathrm{OC}$

$\mathrm{BC} \leq 35$ years (or $\mathrm{BC} \leq 40$ years in case of uninformative family ${ }^{\mathrm{a}}$ )

Bilateral $\mathrm{BC}$ (the first diagnosed $\leq 40$ years)

Triple-negative $\mathrm{BC} \leq 50$ years

High-grade epithelial non-mucinous OC (or fallopian tube or primary peritoneal cancer)

2 or more first degree relatives ${ }^{\mathrm{b}}$ with any combination of the following high-risk features:

Bilateral $\mathrm{BC}+$ another $\mathrm{BC}<50$ years

Male breast cancer

$\mathrm{BC}+\mathrm{OC}$

Two cases of $\mathrm{BC}$ diagnosed before age 50 years

3 or more direct relatives ${ }^{\mathrm{b}}$ with $\mathrm{BC}$ and/or OC:

$\geq 3 \mathrm{BC} \pm \mathrm{OC}$

$B C$ breast cancer, $O C$ ovarian cancer

${ }^{a}$ Less than 2 women who have lived until age 45 or older in each side of the family

b In the same side of the family

\begin{tabular}{|c|c|c|}
\hline & Age & $\begin{array}{l}\text { Evidence and } \\
\text { recommendation }\end{array}$ \\
\hline \multicolumn{3}{|l|}{ Women } \\
\hline Breast self awareness & Starting at age 18 years & IIA \\
\hline $\begin{array}{l}\text { Clinical breast exam every } \\
6-12 \text { months }\end{array}$ & Starting at age 25 years & IIA \\
\hline Annual breast MRI & $25-70$ years & IIA \\
\hline Annual mammogram & $30-35$ to 75 years & IIA \\
\hline $\begin{array}{l}\text { Transvaginal ultrasound and Ca } 12.5 \\
\text { every } 6-12 \text { months }\end{array}$ & 30 years & IIC \\
\hline \multicolumn{3}{|l|}{ Men } \\
\hline Breast self awareness & Starting at age 35 years & IIIC \\
\hline Annual clinical breast exam & Starting at age 35 years & IIIC \\
\hline Basal mammogram & 40 years (individualised) & IIIC \\
\hline Annual Prostate Cancer screening & Starting at age 40 years & IIIB \\
\hline \multicolumn{3}{|l|}{ Men and women } \\
\hline Pancreatic and melanoma & $\begin{array}{l}\text { Consider individualised screening based on } \\
\text { cancers in the family }\end{array}$ & IIIC \\
\hline $\begin{array}{l}\text { Colorectal cancer screening, } \\
\text { especially in BRCA1 }\end{array}$ & $\begin{array}{l}\text { Starting at } 40 \text { years or younger if family } \\
\text { history }\end{array}$ & IIB \\
\hline
\end{tabular}

Table 2 Screening recommendations in BRCA mutation carriers without a personal history of breast cancer might be considered (II,B).

\section{Prophylactic mastectomy}

Retrospective analyses have indicated that bilateral risk reduction mastectomy (BRRM) decreases breast cancer risk by at least $90 \%$ in BRCA1 and BRCA2 mutation carries [10, 14].

In healthy $B R C A 1$ or $B R C A 2$ mutation carriers, BRRM reduces $\mathrm{BC}$ risk when compared to surveillance, while longer follow-up is warranted to confirm survival benefits [15]. 
In a recent prospective analysis, contralateral RRM was found to be associated with improved overall survival in $B R C A 1$ or $B R C A 2$ mutation carriers with a prior primary breast cancer (PBC). Survival benefit was especially seen in young patients ( $<40$ years), with grade $1 / 2$ differentiation and/or no triple-negative phenotype, and in patients not treated with adjuvant chemotherapy [16].

Bilateral risk-reducing mastectomy is an option for healthy $B R C A 1$ and $B R C A 2$ mutation carriers, as well as contralateral mastectomy for young patients with a prior $\mathrm{BC}$ diagnosis (II,B).

\section{Chemoprevention}

Several case-control studies in BRCA mutation carriers with breast cancer show that the use of tamoxifen protects against contralateral breast cancer [17].

Adjuvant tamoxifen reduces the risk of a second breast cancer in patients with a $B R C A$ mutation and a prior $\mathrm{BC}$ (II,A).

In the Breast Cancer Prevention Trial, healthy women with a $B R C A 2$ mutation receiving tamoxifen had a reduction of breast cancer by $62 \%$, an effect not seen among those with a BRCAl mutation, but the overall number of individuals was very small. There are no other chemoprevention trials in $B R C A$ mutation carriers, except for an ongoing randomised clinical trial by the French Federation of Cancer Centers investigating the preventive effect of letrozole in postmenopausal women with a BRCAl or $B R C A 2$ mutation [18]. There is no demonstrated benefit for primary chemoprevention of breast cancer in $B R C A l$ or BRCA2 mutation carriers (I,A).

In a recent meta-analysis of case-control studies in BRCA1 and BRCA2 mutation carriers, a significant $50 \%$ risk reduction of ovarian cancer was associated with the past use of combined oral contraceptives [19]. Nevertheless, there are conflicting results on the effect of oral contraceptive use on breast cancer risk in $B R C A$ mutations carriers. A recent case-control study in BRCAl carriers found that oral contraceptives use before age 25 increases the risk of early onset breast cancer among $B R C A 1$ mutation carriers and the risk increases with duration of use [20].

Use of oral contraceptives protects against ovarian cancer (II,B), but caution should be used when considering them in mutation carriers because of the conflicting results on their effect on breast cancer risk. BRCAl carriers should be advised to avoid oral contraceptive use if the purpose is to prevent ovarian cancer before the age of 25 (IIB).

\section{Treatment strategies in $B R C A$ carriers}

Breast conservative surgery in patients with early stage BC is associated with high rates of second ipsilateral recurrences, but no differences in BC-specific survival compared to mastectomy. Mutation carriers have a higher risk of contralateral BC than sporadic BC patients. Therefore, $B R C A$ genetic testing in patients with early stage $\mathrm{BC}$ may influence their locoregional treatment (III,A).

Platinum salts have shown a high pathological complete response in the neoadjuvant setting among patients with breast cancer and a BRCA1 or BRCA2 germline mutation $[21,22]$. In the metastatic setting, carboplatin has shown a statistically clinical benefit when compared to docetaxel among BRCA mutation carriers [23]. Randomised phase 3 trials of PARPi for patients with $B R C A$-associated $\mathrm{BC}$ are ongoing.

Platinum salts might be considered in the neoadjuvant setting $(\mathbf{I}, \mathbf{C})$, and in the metastatic setting among patients with BC and a BRCA mutation (I,A) (Table 3).

Retrospective studies have shown an improved prognosis, higher response rates and longer treatment-free intervals between relapses in patients with a $B R C A 1 / 2$ mutated ovarian cancer treated with platinum-containing

Table 3 Risk reduction and therapeutic strategies in BRCA mutation carriers

Adjuvant tamoxifen reduces the risk of contralateral breast cancer (IIA)

Benefit of tamoxifen for primary prevention is not demonstrated in BRCA mutation carriers (IA)

Oral contraceptives protect against ovarian cancer (IIB), but caution should be used when considering use of oral contraceptives in mutation carriers because the conflicting results on their effect on breast cancer risk

Bilateral Salpingo-oophorectomy should be recommended between 35 and 40 years and upon completion of child bearing (IA)

Bilateral prophylactic mastectomy reduces the risk of breast cancer by at least $90 \%$ (IIB), and is an option for healthy BRCA1 and BRCA2 mutation carriers, as well as contralateral mastectomy for young patients with a prior breast cancer diagnosis (IIB)

Platinum salts might be considered in neoadjuvant setting (IC) and in the metastatic setting (IA)

PARPi are recommended as maintenance therapy in patients with relapsed platinum-sensitive high-grade serous ovarian cancer (IA) 
regimens compared with sporadic ovarian cancer patients [24]. These tumours also show high sensitivity to anthracyclines [25]. Poly(ADP-ribose) polymerase (PARP) inhibitors lead to synthetic lethality in HR-deficient cells and they are active drugs in patients with BRCA1 or BRCA2mutated ovarian cancer. Olaparib is the first EMA-approved PARPi as a maintenance therapy in patients with relapsed platinum-sensitive high-grade serous ovarian cancer [26].

Alkylating and DNA-damaging agents are recommended for patients with ovarian cancer $(\mathbf{I}, \mathbf{A})$. PARPi are recommended as maintenance therapy in patients with relapsed platinum-sensitive high-grade serous ovarian cancer $(\mathbf{I}, \mathbf{A})$.

\section{Management of women without identified BRCA mutations (BRCAX)}

Women with a breast cancer family history and an inconclusive $B R C A$ genetic test have a higher risk of developing breast cancer $(\mathrm{RR}=3.94,95 \%$ CI 3.09-5.02), but no increased ovarian cancer risk [27].

Monitoring of breast cancer for these women should include breast awareness from age 18 (III,B), clinical breast examination every 6 months since age 25 (III,B) and annual mammography from age 40 or 10 years before the youngest case of breast cancer in the family (II,B). Add an annual breast MRI from age 25 when the $\mathrm{BC}$ lifetime risk is over $20-25 \%$ (IIB). The risk will be determined by predictive models as BRCAPRO, BOADICEA or Tyrer-Cuzyck [28] (Table 4).

Surveillance with ultrasound should not routinely be offered to women at moderate or high risk of $\mathrm{BC}$, but it might be considered when MRI surveillance is not suitable or when results of mammography or MRI are difficult to interpret.

Gynaecological monitoring is not necessary in families with no family history of ovarian carcinoma. If family history of ovarian carcinoma exists, the medical management should be individualised.

Tamoxifen (pre- and post-menopausal women) and raloxifene (only postmenopausal women) are recommended for breast cancer chemoprevention for a maximum of 5 years among women at high risk according to the
NICE guidelines (http://www.nice.org.uk/guidance/cg164), and are approved by the FDA for this purpose. Both drugs can be considered for breast cancer chemoprevention in women $>35$ years at high risk (I,A).

\section{Other hereditary breast cancer syndromes}

Other breast cancer susceptibility genes have been identified as TP53, PTEN and PALB2 as high penetrance, and CHEK2, BRIP1, ATM, BARD1, NBN, RAD51C with lower penetrance [2]. $C D H 1$ is considered high-penetrance gene for diffuse gastric cancer, but the BC risk has not been fully established.

TP53 is involved in only $1 \%$ of hereditary breast cancer cases. It is associated with a high lifetime risk of cancer, most diagnosed at young age as bone and soft tissue sarcoma, premenopausal breast cancer, acute leukaemia, colon cancer, adrenal cortex carcinoma, brain tumours and ovarian cancer.

Breast screening must begin at age 20 years with annual MRI and add annual mammogram at age 30 . The option of risk-reducing mastectomy should be discussed in TP53 mutation carriers (II,A).

Cowden syndrome $(P T E N)$ is a rare hereditary cancer syndrome. The incidence is 1 in 200.000 . The estimated cumulative lifetime risk for breast cancer is $67-85 \%$ and patients may develop cancers of thyroid, endometrial, colorectal, renal and melanoma. Hamartomatous lesions are characteristic manifestations of this syndrome.

Breast screening consists of annual mammogram and breast MRI beginning at age 30. The option of risk-reducing mastectomy and hysterectomy should be discussed (II,A).

Germline mutations in $\mathrm{CDHI}$ are associated with Hereditary Diffuse Gastric Cancer Syndrome [29]. Women have a cumulative lifetime risk of lobular breast cancer of 23-68\%. CDH1 germline mutations can also be found in the absence of DG cancer, especially in families with $>3$ lobular BC or bilateral lobular cancer before age 50 .

Annual mammography and breast MRI from the age of 35 is recommended in $\mathrm{CDH1}$ mutation carriers (II,A).

The breast cancer risk associated with mutations in the lower-penetrance genes is currently imprecise. Clinical management is case-by-case depending on the family
Table 4 Surveillance in women from high-risk families without identified BRCA mutations
Breast self awareness starting at age 18 (IIIB)

Semiannual clinical breast exam starting at age 25 (IIIB)

Annual mammogram starting at age 40-70, or 10 years before the youngest age of BC in the family (IIB) If lifetime risk $>20-25 \%$, consider annual breast MRI starting at age 25 (IIB)

Ovarian early detection is not necessary in women with no family history of ovarian carcinoma (IIA) 
history [30]. The use of multigene panel testing will increase the diagnosis of such mutations, but more clinical research is needed to learn about their precise clinical meaning.

Evidence levels To assign a level of evidence and a grade of recommendation to the different statements of this guideline, it was decided to use the Infectious Diseases Society of America-US Public Health Service Grading System for Ranking Recommendations in Clinical Guidelines to determine the quality of evidence and strength of recommendation in each of the consensus recommendations [31].

Acknowledgments Joan Brunet benefits from a RTICC Grant RD 12/0036/0053.RD12/0036/008.

\section{Compliance with ethical standards}

Conflict of interest The authors declare that they have no conflict of interest.

Open Access This article is distributed under the terms of the Creative Commons Attribution 4.0 International License (http://crea tivecommons.org/licenses/by/4.0/), which permits unrestricted use, distribution, and reproduction in any medium, provided you give appropriate credit to the original author(s) and the source, provide a link to the Creative Commons license, and indicate if changes were made.

\section{References}

1. Mavaddat N, Peock S, Frost D, Ellis S, Platte R, Fineberg E, et al. Cancer risks for $B R C A 1$ and $B R C A 2$ mutation carriers: results from a prospective analysis of EMBRACE. J Natl Cancer Inst. 2013;105(11):812-22.

2. Chen S, Parmigiani G. Meta-analysis of $B R C A 1$ and $B R C A 2$ penetrance. J Clin Oncol. 2007;25:1329-33.

3. Easton DF, Pharoah PDP, Antoniou AC, Tischkowitz M, Tavtigian SV, Nathanson KL, et al. Gene-panel sequencing and the prediction of breast-cancer risk. N Engl J Med. 2015;372:2243-57.

4. Alsop K, Fereday S, Meldrum C, deFazio A, Emmanuel C, George J, et al. BRCA mutation frequency and patterns of treatment response in BRCA mutation-positive women with ovarian cancer: a report from the Australian Ovarian Cancer Study Group. J Clin Oncol. 2012;30:2654-63.

5. Couch F, Hart SN, Sharma P, Toland AE, Wang X, Miron P, et al. Inherited mutations in 17 breast cancer susceptibility genes among a large triple-negative breast cancer cohort unselected for family history of breast cancer. J Clin Oncol. 2015;33:304-11.

6. Desmond A, Kurian AW, Gabree A, Mills MA, Anderson MJ, Kobayashi Y, et al. Clinical actionability of multigene panel testing for hereditary breast and ovarian cancer risk assessment. JAMA Oncol. 2015;. doi:10.1001/jamaoncol. 2015.2690 .

7. Phi X-A, Houssami N, Obdeijn IM, Warner E, Sardanelli F, Leach MO, et al. Magnetic resonance imaging improve breast screening sensitivity in BRCA mutation carriers age $\geq 50$ years: evidence from an individual patient data metaanalysis. J Clin Oncol. 2015;33:349-56.

8. Saadatmand S, Obdeijn IM, Rutgers EJ, Oosterwijk JC, Tollenaar RA, Woldringh GH, et al. Survival benefit in women with BRCA1 mutation or familial risk in the MRI screening study (MRISC). Int J Cancer. 2015;137:1729-38.

9. Bancroft EK, Page EC, Castro E, Lilja H, Vickers A, Sjoberg D, et al. Targeted prostate cancer screening in BRCA1 and BRCA2 mutation carriers: results from the initial screening round of the IMPACT study. Eur Urol. 2014;66(3):489-99.

10. Domchek SM, Friebel TM, Singer CF, Evans DG, Lynch HT, Isaacs C, et al Association of risk-reducing surgery in $B R C A 1$ or $B R C A 2$ mutation carriers with cancer risk and mortality. JAMA. 2010;304:967-75.
11. Finch AP, Lubinski J, Moller P, Singer CF, Karlan B, Senter L, et al. Impact of oophorectomy on cancer incidence and mortality in women with a BRCA1 or BRCA2 mutation. J Clin Oncol. 2014;32:1547-53.

12. Rebbeck TR, Kauff ND, Domchek SM. Meta-analysis of risk reduction estimates associated with risk-reducing salpingo-oophorectomy in BRCAl or BRCA2 mutation carriers. J Natl Cancer Inst. 2009;101:80-7.

13. Rebbeck TR, Friebel T, Wagner T, Lynch HT, Garber JE, Daly MB, et al. Effect of short-term hormone replacement therapy on breast cancer risk reduction after bilateral prophylactic oophorectomy in BRCA1 and BRCA2 mutation carriers: the PROSE Study Group. J Clin Oncol. 2005;23(31):7804-10.

14. Hartmann LC, Sellers TA, Schaid DJ, Frank TS, Soderberg CL, Sitta DL, et al. Efficacy of bilateral prophylactic mastectomy in BRCA1 and BRCA2 gene mutation carriers. J Natl Cancer Inst. 2001;93:1633-7.

15. Heemskerk-Gerritsen BA, Menke-Pluijmers MB, Jager A, Tilanus-Linthorst MM, Koppert LB, Obdeijn IM, et al. Substantial breast cancer risk reduction and potential survival benefit after bilateral mastectomy when compared with surveillance in healthy BRCA1 and BRCA2 mutation carriers: a prospective analysis. Ann Oncol. 2013;24:2029-35.

16. Heemskerk-Gerritsen BA, Rookus MA, Aalfs CM, Ausems MG, Collée JM, Jansen $\mathrm{L}$, et al. Improved overall survival after contralateral risk-reducing mastectomy in BRCA1/2 mutation carriers with a history of unilateral breast cancer: a prospective analysis. Int J Cancer. 2015;136:668-77.

17. Phillips KA, Milne RL, Rookus MA, Daly MB, Antoniou AC, Peock S, et al Tamoxifen and risk of contralateral breast cancer for BRCA1 and BRCA2 mutation carriers. J Clin Oncol. 2013;31:3091-9.

18. Bonanni B, Lazzeroni M. Acceptability of chemoprevention trials in high-risk subjects. Ann Oncol. 2013;24(Sup 8):viii42-6.

19. Cibula D, Zikan M, Dusek L, Majek O. Oral contraceptives and risk of ovarian and breast cancers in $B R C A$ mutation carriers: a meta-analysis. Expert Rev Anticancer Ther. 2011;11(8):1197-207.

20. Kotsopoulos J, Lubinski J, Moller P, Lynch HT, Singer CT, Eng C, Hereditary Breast Cancer Clinical Study Group, et al. Timing of oral contraceptive use and the risk of breast cancer in BRCA1 mutation carriers. Br Can Res Treat. 2014;143(3):579-86.

21. Telli ML, Jensen KC, Vinayak S, Kurian AW, Lipson JA, Flaherty PJ, et al. Phase II study of gemcitabine, carboplatin, and iniparib as neoadjuvant therapy for triple-negative and BRCA1/2 mutationassociated breast cancer with assessment of a tumor-based measure of genomic instability: PrECOG 0105 J Clin Oncol. 2015;33(17):1895-901.

22. von Minckwitz G, Hahnen E, Fasching PA, Hauke J, Schneeweiss A, Salat C, et al. Pathological complete response (pCR) rates after carboplatin-containing neoadjuvant chemotherapy in patients with germline BRCA (gBRCA) mutation and triple-negative breast cancer (TNBC): results from GeparSixto. J Clin Oncol. 2014;32(5s):1005.

23. Tutt A, Ellis P, Kilburn LS, Gilett C, Pinder S, Abraham J, et al. TNT: a randomized phase III trial of carboplatin (C) compared with docetaxel (D) for patients with metastatic or recurrent locally advanced triple negative or BRCA1/ 2 breast cancer CRUK/07/012). Cancer Res Suppl. 2014;75:S3-01.

24. Tan DS, Kaye SB. Chemotherapy for patients with BRCA1 and BRCA2-mutated ovarian cancer: same or different? Am Soc Clin Oncol Educ Book. 2015;35:114-21.

25. Kaye SB, Lubinski J, Matulonis U, Ang JE, Gourley C, Karlan BY, et al. Phase II, open-label, randomized, multicenter study comparing the efficacy and safety of olaparib, a poly (ADP-ribose) polymerase inhibitor, and pegylated liposomal doxorubicin in patients with BRCA1 or BRCA 2 mutations and recurrent ovarian cancer. J Clin Oncol. 2012;30(4):372-9.

26. Ledermann J, Harter P, Gourley C, Friedlander M, Vergote I, Rustin G, et al. Olaparib maintenance therapy in patients with platinum-sensitive relapsed serous ovarian cancer: a preplanned retrospective analysis of outcomes by BRCA status in a randomised phase 2 trial. Lancet Oncol. 2014;15(8):852-61.

27. Metcalfe KA, Finch A, Poll A, Horsman D, Kim-Sing C, Scott J, et al. Breast cancer risks in women with a family history of breast or ovarian cancer who have tested negative for a BRCA1 or BRCA2 mutation. $\mathrm{Br} \mathrm{J}$ Cancer. 2009;100(2):421-5.

28. Warner E, Messersmith H, Causer P, Eisen A, Shumak R, Plewes D. Systematic review: using magnetic resonance imaging to screen women at high risk for breast cancer. Ann Intern Med. 2008;148(9):671-9.

29. Van der Post RS, Vogelaar IP, Carneiro F, Guilford P, Huntsman D, Hoogerbrugge $\mathrm{N}$, et al. Hereditary diffuse gastric cancer: updated clinical guidelines with emphasis on germline CDH1 mutation carriers. J Med Genet. 2015;52:361-74.

30. National Comprehensive Cancer Network (NCCN). NCCN Clinical Practice guidelines in oncology. http://www.nccn.org/professionals/physician_gls/f_ guidelines.asp, 2015.

31. Khan AR, Khan S, Zimmerman V, Baddour LM, Tleyjeh IM. Quality and strength of evidence of the infectious diseases society of America Clinical Practice Guidelines. Clin Infect Dis. 2010;51(10):1147-56. 